\title{
La influencia de la música y el ejercicio físico en la preparación física y psicológica
}

\section{Álvaro Carrasco Valdayo}

\section{Graduado en Ciencias de la Actividad Física y del Deporte (Universidad de Huelva)}

\section{Email: alvarocarrasc079@gmail.com}

\section{RESUMEN:}

El comportamiento de la música en variables físicas y psicológicas es un campo no consolidado actualmente en el marco de la literatura científica. El propósito de este trabajo fue evaluar la influencia de la música en la preparación física y psicológica de una muestra de sujetos sedentarios con edades comprendidas entre 25 y 30 años $(M=27.5)$. Se utilizó un diseño cuasi-experimental donde la muestra $(N=40)$ se dividió en dos grupos (experimental y control) que se sometieron a un programa de mejora de la condición física. El grupo experimental $(n=$ $20)$ en el que se empleó un programa con presencia de música y el grupo control ( $n=20)$ que utilizo el mismo programa pero sin música. Las variables físicas estudiadas fueron la potencia aeróbica y anaeróbica, evaluadas mediante pre-test y post-test. Por otro lado, las variables psicológicas fueron el carácter de esfuerzo, la intención de seguir practicando ejercicio físico, las emociones y la motivación, todas evaluadas durante la intervención. Los resultados mostraron mejoras significativas en ambos grupos en las variables físicas, siendo más amplias en el grupo experimental, mientras que el comportamiento de las variables psicológicas fue condicionado por la música, mostrándose un menor carácter de esfuerzo, una mayor intención de seguir haciendo ejercicio y una mayor motivación en canciones con tempo elevado (> 100 bpm). Así pues, la música parece influir tanto en la preparación física como psicológica de los sujetos.

PALABRAS CLAVE: Condición Física, Intervención, Tempo Musical 


\section{INTRODUCCIÓN}

La actividad física y la música han estado muy unidas durante toda la historia de la humanidad. Las primeras tribus indígenas ya la usaban en sus rituales o formas de supervivencia como correr y cazar (Mandianes, 2003). No obstante, la influencia de la música en la preparación física y psicológica de las personas es un campo poco explorado en el marco de la literatura científica, aunque actualmente, se está incrementando cada vez más el interés por este tipo de investigaciones dada la efectividad de los resultados en el ámbito de la actividad física y el deporte (Karageorghis y Priest, 2012).

La música ha sido definida como una de las bellas artes que combina sonidos con vistas a la belleza de su forma y la expresión de emociones (Murray, Sujan, Hirt, y Sujan, 1990). Umemoto (1990) la define de forma más simple; "El arte del sonido". La música se puede catalogar como un conjunto de factores clasificados como estilo, idioma, ritmo, tempo, melodía, armonía e intensidad (Bunt y Stige, 2014; Del Olmo, 2009; Palomares, 2014), que pueden afectar en forma conjunta o independiente en la interpretación de los resultados de las variables a medir. Esto ha provocado resultados contradictorios que imposibilitan tener una conclusión clara de los verdaderos efectos de la música en las personas (Karageorghis y Terry, 1997).

Unos de los conceptos con mayor relevancia a tener en cuenta en nuestro estudio será el tempo de la música. Caballero-Meneses y Menez (2010) lo definen como aquello que indica la velocidad de la música. Se mide en beats por minuto (bpm) y se caracteriza por establecer cantidades equidistantes de pulsaciones por minuto ( 80 bpm; 80 pulsaciones en 60 segundos).

Actualmente, hay evidencias de que la música se puede usar para mejorar la memoria, la comunicación, las relaciones interpersonales (Sánchez, 2010) para mejorar el ritmo respiratorio, los problemas del aprendizaje (Montánchez, 2012), la imaginación, la observación y la agilidad mental (Elvira, 2004), también para tratar diversas psicopatologías como la depresión (Dueñas y Herrera, 2015), o aumentar el bienestar en personas con Síndrome de Down (Pineda y Pérez, 2011). En este sentido, la música puede ser considerada como un tipo de "droga legal" que mejora las facultades del ser humano (Karageorghis y Priest, 2012), por ello pretendemos indagar en este estudio el uso de la misma en el ámbito de la actividad física y del deporte.

\subsection{Música y carácter de esfuerzo percibido}

Este posible efecto parece poder explicarse según las teorías de "percepción selectiva" (Broadbent, 1958) y de "atención focalizada" (Hernández-Peon, 1961), según las cuales el sistema nervioso puede atender únicamente a un estímulo (musical, en este caso) a expensas de discriminar el resto de estímulos envolventes (Copeland y Franks, 1991; Nideffer, 1976) atenuando la sensación de cansancio en el individuo (Guillén y Ruiz-Alfonso, 2013; Hernández-Peon, 1961; Ju-Han y Jing-Horng Lu, 2013; Pennebaker y Lightner, 1980). Rejeski (1985) también señala que la capacidad del sistema nervioso central es limitada y que solo es capaz de atender a un estímulo, por lo que, cuando una actividad física es acompañada por música, los estímulos que produce ésta pueden evitar las señales de retroalimentación relacionadas con el esfuerzo físico (Karageorghis y Priest, 2012). De la misma forma, Hernández-Peón (1961) indicó que la música podía producir estímulos placenteros en una vía sensorial a la vez que inhibía la actividad eléctrica y, por lo tanto, la transmisión de información, en otra vía. 
Rejeski (1985) afirma que la intensidad del ejercicio está relacionada con la capacidad de la música para inhibir el procesamiento de otros estímulos sensoriales. Dicho autor hace referencia a que en actividades de intensidad elevada, las señales fisiológicas son las que dominan la capacidad de procesamiento, mientras que en ejercicios de niveles moderados, ambas señales (internas del propio cuerpo y externas como la música), pueden ser procesadas en paralelo. Por lo tanto, aunque la música no puede cambiar la fatiga producida al realizar ejercicios intensos, sí puede cambiar la percepción del sujeto acerca de ésta. Este efecto es mayor sedentarios (Karageorghis y Terry, 1997).

\subsection{Música y motivación}

El uso de la música en diferentes contextos de actividad física puede estar sustentado teóricamente por el modelo jerárquico de motivación intrínseca y extrínseca de Vallerand (2001), ya que puede influir en el tipo de motivación experimentada (Karageorghis y Terry, 1997). Parece existir la tendencia de que la música mejora la motivación intrínseca, ya que promueve el disfrute de la actividad, sin embargo, no hay evidencias actuales de que la música promueve la motivación extrínseca (Brooks y Brooks, 2010; Karageorghis y Terry, 1997).

Karageorghis, Terry y Lane (1999) mencionaron cuatro factores que dotaban de cualidades motivacionales una pieza musical: el tempo (bpm), la musicalidad (armonía y melodía), el impacto cultural y la asociación de la música con la evocación de imágenes extra-musicales. Posteriormente, Karageorghis y Priest (2012), consideran el tempo como el factor más importante, por ello será el elemento con más peso en esta investigación.

\subsection{Música y condición física}

Diversos estudios hablan de la importancia de la música en la mejora de la condición física. Karageorghis y Terry (1997) hacen referencia a la predisposición innata del organismo para sincronizar el movimiento con el componente rítmico de la música. Para situarnos, MacDougal en 1903 ya identificó la relación entre el tempo musical y las formas naturales de movimiento (caminar y correr). Sin embargo, Bonny (1987) relaciona el tempo musical con la periodicidad del organismo; activación de las neuronas, respiración, latidos del corazón, ciclo de sueño, etc.

A raíz de esto, hay numerosas evidencias sobre el aumento del trabajo físico submáximo, si éste puede sincronizarse con el tempo de una música, lo que conllevaría a una mejora de la condición física (Waterhouse, Hudson, y Edwards, 2010). Durante un ejercicio físico submáximo continuo el organismo tiende a responder al elemento rítmico de la música dando como resultado una sincronización entre el tempo de la música y la acción del movimiento (Karageorghis y Terry, 1997). Estos efectos fueron demostrados en pruebas como el "Test de escalón del Forest Service" (Hayakawa, Miki, Takada, y Tanaka 2000), cicloergometría (Anshel y Marisi, 1978) ejercicios de calistenia (Uppal y Datta, 1990), 400 metros lisos (Simpson y Karageorghis, 2006) o ejercicios de carácter aeróbico (Van der Vlist, Bartneck, y Mäueler, 2011).

\subsection{Música y emociones}

Para conocer la incidencia que tiene la música en la regulación de las emociones es conveniente aproximarnos a la terminología existente. Por ello, para distinguir una emoción de un estado de ánimo usaremos el criterio de la duración 
(Reeve, 2003). Según dicho autor la emoción presenta una duración corta (segundos o minutos), mientras que los estados de ánimo suelen durar horas. Por lo tanto, el uso de piezas musicales breves implica que la dimensión afectiva medida es la emotiva, no la anímica (Caballero-Meneses y Menez, 2010).

Hay autores que clasifican la música en función de las emociones que se asocian a las mismas. Lipschultz y Chambliss (1992) la clasifica en función del tempo musical:

- "Música positiva": música con tempo alto. Sensaciones descritas de felicidad, inspiración, satisfacción y bienestar.

- "Música negativa": Música con tempo lento. Sensaciones más asociadas a tristeza, insatisfacción, desconfianza y falta de motivación.

Otra clasificación es la de Karageorghis et al. (1996) quienes la dividen en dos tipos; "música estimulante" (tempo >130 bpm) y "música relajante" (tempo <90 bpm). Pero no siempre las emociones afectan de la misma forma en el ejercicio físico. Hanin (2003) afirma que tanto las emociones positivas como negativas pueden resultar funcional o disfuncional para cada individuo. Por ejemplo, emociones negativas como la ira pueden facilitar el desempeño de un individuo en un entrenamiento de resistencia intenso (funcional), sin embargo, tendría poca utilidad en el contexto de una clase de yoga (disfuncional).

Szmedra y Bacharach propusieron en 1998 que estados psicológicos evocados por la música durante el ejercicio como el alivio de la ansiedad, puede contribuir a la vasodilatación, lo que influye en las variables hemodinámicas, el aclaramiento de ácido láctico y la disminución del cortisol, que es un indicador de emociones negativas (John, Verma, y Khanna, 2010). Por lo tanto, los efectos psicológicos de la música durante el ejercicio pueden influir a nivel fisiológico.

Otro de los factores condicionantes en las emociones es el tempo de la música, aunque la influencia de éste sobre las emociones puede estar limitada por la personalidad, especialmente en la extroversión e introversión del sujeto (Larsen y Buss, 2005). Ambos autores afirman que los introvertidos son más rápidamente excitables que los extrovertidos cuando se incrementan la velocidad de las piezas musicales. Por otra parte, la reacción del individuo es distinta si a la música se le atribuye una asociación extra-musical (Gfeller, 1988) por ejemplo; la banda sonora de la película Rocky (tempo bajo) ante un ejercicio intenso.

\subsection{Música y adherencia a la práctica deportiva}

La música contribuye un elemento de disfrute para la realización de actividad física, lo que podría aumentar la adherencia (Gluch, 1993; Mann, 1979; Karageorghis y Priest, 2012). Del mismo modo, se postula que las correspondientes emociones positivas provocadas por la música podría incrementar la adherencia al ejercicio (Karageorghis, 1999), aunque esto también dependerá de las preferencias musicales de los sujetos (García, 2004).

A pesar de la existencia de investigaciones sobre la influencia de la música en el comportamiento humano, en este trabajo se han relacionado variables psicológicas con físicas en un contexto de personas sedentarias y analizando el efecto del tipo de música en cada una de ellas. Por lo que esta combinación resulta original dada la escasa literatura científica que estudie la relación entre todas las variables objeto de estudio. En este sentido, el objetivo principal de este trabajo fue evaluar la influencia de la música en la preparación física y psicológica de una muestra de sujetos sedentarios sometidos a un programa de ejercicio físico similar. 
Asimismo, a través del presente trabajo se pretende intervenir con dos programas de mejora de condición física (en adelante CF), uno con presencia de música y otro sin ella para conocer su influencia en la preparación física (potencia aeróbica y anaeróbica) de los participantes. Por lo que se hipotetizó que con la intervención de un programa de mejora de la CF con presencia de música se obtendrá mejoras más amplias en los participantes en comparación con aquellos que lo realizan sin música.

Otro objetivo específico fue analizar la influencia de la música en el carácter de esfuerzo percibido (CEP). Por un lado, se pretende valorar las diferencias entre el CEP final de los participantes del programa de mejora de la CF con y sin música. En esta línea, surge la hipótesis de que los participantes del programa de mejora de la CF con música tendrán un carácter de esfuerzo percibido (CEP) mayor que los del programa sin música. Por otro lado, se pretende identificar el efecto de los distintos tipos de música en el CEP de los sujetos del programa de CF con música. Se prevé que con la música de tempo elevado (>100 bpm) tendrán un CEP menor que con la música de tempo bajo (<100 bpm).

También se persigue medir el comportamiento de la adherencia a la práctica deportiva de los participantes de ambos programas una vez finalizada la intervención. Por ello, se hipotetizó que los sujetos del programa de mejora de la CF con música tendrán una mayor intención de mantenerse físicamente activo con respecto a los del programa sin música. Además, se pretende comprobar el efecto de los distintos tipos de música en las emociones percibidas de los sujetos, profundizando más concretamente en el comportamiento de la motivación. De modo que con la música de tempo elevado (>100 bpm) se identificarán un mayor número de emociones positivas, mientras que con la música de tempo bajo (<100 bpm) se apreciarán mayores emociones negativas. Y por último, se apreciará una mayor motivación en canciones con tempo musical elevado (>100 bpm) en comparación con las de tempo musical bajo $(<100 \mathrm{bpm})$.

\section{MÉTODO}

\subsection{Diseño}

Se llevó a cabo un diseño cuasi-experimental donde se recogió una información inicial de la muestra a modo de pre-test, se siguió evaluando durante 3 meses de intervención y finalmente se empleó un post-test para comprobar la evolución de los resultados. Como variables independientes se eligieron el tipo de música (en función del tempo musical; alto/bajo) y el tipo de programa de mejora de la condición física (con/sin música). Como variable dependiente así como objeto de estudio, se seleccionaron; la condición física, la intención de seguir haciendo ejercicio, el carácter de esfuerzo percibido, las emociones percibidas (positivas/negativas) y la motivación. Además, se controló el cumplimiento del programa así como posibles problemas personales de los participantes. Asimismo, se trató de minimizar los efectos de la variable meteorológica, eligiendo días con temperaturas y viento similares tanto en el pre-test como en el post-test.

\subsection{Participantes}

El estudio empleó un muestreo no probabilístico intencional siguiendo unos criterios de inclusión específicos (frecuencia actividad física, edad, residencia y gusto 
por la música). Por ello se escogió una muestra de 40 personas sedentarias de la localidad de Bollullos Par del Condado (Huelva), pertenecientes al club de ajedrez de la localidad, con edades comprendidas entre 25-30 años $(M=27.5)$ y que le gusten y suelan escuchar música frecuentemente. Dicha muestra fue subdividida en dos grupos: un grupo experimental (GE) de 20 sedentarios/as sometidos/as a un programa de mejora de condición física con presencia de música (10 hombres y 10 mujeres), y un grupo de control (GC) de 20 sedentarios/as sometidos/as al mismo programa de mejora de condición física sin presencia de música (10 hombres y 10 mujeres).

\subsection{Instrumentos}

Como entrevista inicial, se llevó a cabo una serie de preguntas elaboradas para conocer el gusto de los participantes por la música así como la regularidad con la que la escuchan. En dicha entrevista, para escoger una muestra de personas sedentarias como criterio de inclusión, se llevó a cabo la versión corta del Cuestionario Internacional de Actividad Física (IPAQ) que nos proporciona información sobre el tiempo empleado al caminar, en actividades de intensidad moderada, vigorosa y en actividades sedentarias.

Para medir la potencia aeróbica máxima de los sujetos se empleó el Test de Course Navette. Según García y Secchi (2014) la prueba se basa en correr el mayor tiempo posible entre dos líneas separadas por $20 \mathrm{~m}$ en doble sentido. El ritmo de carrera es impuesto por una señal sonora o "beep". Las primeras etapas son de velocidad baja y tienen como objetivo familiarizarse con el test y, a su vez, realizar una entrada en calor específica (García y Secchi, 2014). Siguiendo con la línea de estos autores, el sujeto debe pisar detrás de la línea de 20 metros en el momento justo en que se emite la señal sonora. El test finaliza cuando el sujeto se detiene porque alcanzó la fatiga o cuando por dos veces consecutivas no llega a pisar detrás de la línea al sonido del "beep".

Para evaluar la potencia anaeróbica de los sujetos empleamos el test de Burpee (Campos y Ramón, 2006). La prueba consiste en realizar una serie de ejercicios que combinan tanto el trabajo de tren inferior y superior de forma continua durante 1 minuto (Campos y Ramón, 2006). Por ello se contabiliza el número de veces que el sujeto es capaz de realizar un ejercicio determinado.

En cuanto a la música, se seleccionaron los géneros musicales que más solicitaron los participantes en la entrevista inicial, tratando de combinar canciones con distinto tempo. Por lo que se usaron en gran parte de la intervención el género house en casi todas sus ramas (deep house, progressive house, electro house, etc.). Además, otros géneros como el rock, pop, techno, drum and bass o incluso la música clásica también tuvieron protagonismo. Las canciones se agruparon en listados en función del tempo deseado, las cuales tenían que ser escuchadas por los participantes del GE mientras realizaban sus ejercicios.

El programa de mejora de la CF estaba formado por un conjunto de ejercicios orientados hacia la mejora de la potencia aeróbica y anaeróbica de los individuos. Constaba de sesiones de 60 minutos aproximadamente (5-6 días por semana) y ejercicios con una carga de tipo incremental; primeras 5 semanas (mismo volumen e intensidad) y semanas restantes (incrementando progresivamente $50-80 \%$ Fcmax).

Para conocer en qué grado la música que escucha el sujeto le motiva durante el ejercicio físico, se tradujo al castellano y se usó el cuestionario "The Brunel Music Rating Inventory-3" (BMRI-3) de Karageorghis (2008). El instrumento consiste en escuchar diversas piezas musicales mientras se realiza el ejercicio físico. Una vez 
acabado, el sujeto indica en qué medida está de acuerdo con los seis ítems relacionados con el grado de motivación de la música escuchada. Para ello se usa una escala tipo Likert donde se pide que califique de 1 a 7 su grado de acuerdo con la misma.

Para medir el esfuerzo percibido por el individuo en función el tipo de ejercicio que realiza se empleó la escala de Borg (1982). El concepto del esfuerzo percibido es una valoración subjetiva que indica la opinión del sujeto respecto a la intensidad del trabajo realizado (Morgan, 1973). Se le pide al sujeto que asigne un número del 6 al 20 después del ejercicio para representar la sensación subjetiva de la cantidad de trabajo desempeñado.

Para conocer las emociones manifestadas en función del tipo de música escuchada se adaptó la versión traducida al castellano del cuestionario "Profile of Mood States" (POMS) de Andrade, Arce, De Francisco, Torrado y Garrido (2013). EI cuestionario utilizado está compuesto por 7 ítems que los participantes tienen que responder siguiendo un formato tipo Likert de 1 (Bastante en desacuerdo) a 7 (Bastante de acuerdo). Para la clasificación de las emociones se ha tenido en cuenta la propuesta por Lazarus (2000) y Bisquerra (2000); emociones positivas (alegría, bienestar, confianza, felicidad), emociones negativas (nerviosismo, ansiedad, agresividad, tristeza, indecisión, confusión).

Para estimar la adherencia a la práctica deportiva se usó una adaptación de la de la Medida de la Intencionalidad para ser Físicamente Activo (MIFA; Moreno, Moreno y Cervelló, 2007), que es la versión en español del "Intention to be Physically Active" de Hein, Müür y Koka (2004). Este cuestionario está compuesto de cinco ítems para medir la intención del sujeto de ser físicamente activo. Las respuestas corresponden a una escala tipo Likert entre 1 (Totalmente en desacuerdo) y 5 (Totalmente de acuerdo).

Por último, para identificar la velocidad del tempo musical de cada una de las canciones en beats por minuto (bpm) nos apoyamos en un software editor específico llamado "MixMeister Fusion". Al seleccionar la canción deseada, nos identifica automáticamente la duración así como la velocidad de la misma en bpm.

\subsection{Procedimiento}

En cuanto a la cronología de los acontecimientos, antes de comenzar con la recogida de datos, se firmó un consentimiento informado por parte de 59 personas pertenecientes al club de ajedrez, ya que por conocimiento previo, se caracterizaba por ser un conjunto de personas aparentemente inactivas físicamente. Acto seguido se realizaron entrevistas con cada participante para conocer la regularidad con la que realizan actividad física. Se empleó la versión corta del Cuestionario Internacional de Actividad Física (IPAQ) para incluir en el estudio únicamente a los sedentarios/as. Este cuestionario excluyó a 19 personas que no reunían las características exigidas. Al mismo tiempo, también se entregó una ficha con una serie de preguntas elaboradas para conocer el gusto por la música de los sujetos así como la regularidad con la que éstos la escuchan. Días posteriores, se analizaron los datos iniciales obtenidos y se formaron los grupos mixtos (GE y GC) en función de la frecuencia de actividad física (sedentario/a) así como el gusto y la regularidad con la que escuchan música.

A modo de pre-test, se tomó una primera medida para conocer el estado de la condición física de los participantes de ambos grupos. Se realizaron dos pruebas llevadas a cabo por el autor del trabajo y la colaboración de 5 voluntarios; una para la medición de la potencia anaeróbica (Test de Burpee) y otra para conocer el desarrollo 
de la potencia aeróbica (Test de Course Navette). Ambas fueron realizadas al aire libre, más concretamente en el espacio central del "Velódromo Municipal" de Bollullos Par del Condado, que se caracteriza por ser un terreno llano y de asfalto. Los test se emplearon uno tras otro en el orden mencionado, con un período de descanso de 10 minutos entre cada uno. Al finalizar, se les facilitó a los participantes de ambos grupos el mismo listado de ejercicios semanales. Además, se entregó al GE un listado extra elaborado de canciones para que las compaginaran con sus entrenamientos.

Durante las 5 primeras semanas se trató de observar las sensaciones que producía los distintos tipos música en los participantes del GE, por lo que durante dicho período, ambos grupos realizaron la misma rutina de ejercicios. En estas primeras 5 semanas, el GE escuchó listados de música con tempos musicales diferentes; Semana 1 (100-120 bpm), Semana 2 (80-100 bpm), Semana 3 (110-130 bpm), Semana 4 (<80 bpm) y Semana 5 (>130 bpm). A partir de la 5a semana, viendo que las canciones con tempo musical elevado eran las que proporcionaba mayor motivación y emociones positivas, se elaboró un nuevo listado de canciones con tempo (>100 bpm) para que la escucharan en sus entrenamientos. Por ello se emplearon ejercicios con cargas de tipo incremental (volumen e intensidad) para ambos grupos con vistas a mejorar la condición física de los participantes y así poder apreciar si las mejoras en el GE son más acentuadas que en las del GC.

En estas primeras 5 semanas nos encargamos de tener controladas las variables objeto de estudio a través de entrevistas personales con los participantes de ambos grupos en la sede del club de ajedrez o vía telefónica cada semana. De esta forma, se emplearon los cuestionarios BMRI-3 de Karageroghis (2008), la adaptación del POMS (Andrade et al., 2013) y la Escala de Borg para conocer el esfuerzo percibido de los entrenamientos semanales. Además de los instrumentos utilizados, tuvimos en cuenta factores como la regularidad o complicaciones que les pudieran surgir para completar el programa. Asimismo, desde la $5^{\underline{a}}$ semana hasta la $12^{a}$, el seguimiento fue menos exhaustivo, asegurándonos que estaban cumpliendo el protocolo establecido adecuadamente.

Una vez acabado el período de intervención (12 $2^{\mathrm{a}}$ semana), se llevó a cabo el post-test para comprobar la evolución que ha tenido la potencia aeróbica y anaeróbica. Y por último, se usó la adaptación del MIFA (Moreno et al., 2007) en ambos grupos para conocer la intención de seguir practicando ejercicio físico, así como la Escala de Borg para determinar el esfuerzo percibido final por los participantes en función del programa de A.F. desarrollado.

\subsection{Análisis de datos}

En primer lugar, se comparó la toma pretest del grupo experimental (GE) y del grupo control (GC) de los valores de CF (potencia aeróbica y pontencia anaeróbica) para analizar si existían diferencias significativas antes de la intervención entre ambos grupos. A continuación, para comprobar las diferencias intra-grupo entre la toma pretest y la postest se utilizó una prueba t para muestras relacionadas con cada uno de los grupos (GE y GC). De forma descriptiva, se comentaron las diferencias intergrupo. Al finalizar la intervención, se analizaron las diferencias entre el GE y el GC en las variables: esfuerzo percibido e intención de seguir siendo activo. Asimismo, se compararon los promedios dentro del GE de las variables (motivación, esfuerzo percibido y adherencia) entre las semanas con música de tempo elevado ( $1^{\circ}, 3^{\circ}$ y $5^{\circ}$ ) y de tempo bajo $\left(2^{\circ}\right.$ y $\left.4^{\circ}\right)$ a través de pruebas $T$ para la misma muestra. Para ello, los datos fueron analizados mediante el software SPSS (v. 20.0). 


\section{RESULTADOS}

\subsection{Análisis preliminares}

En lo referente a las características de los participantes de cada grupo, se relacionaron los resultados de las variables físicas obtenidos en el pre-test. No presentaron diferencias significativas; promedio Test Course Navette (GE $=26.04$ y GC = $26.48)$, promedio Test de Burpee $(\mathrm{GE}=23.85$; $\mathrm{GC}=23.80)$. Por lo que ambos grupos partían de un estado de forma similar.

\subsection{Efectos de la intervención (GE y GC)}

\subsubsection{Pre-test/post-test condición física}

En cuanto a las pruebas para la evaluación de la condición física, hubo mejoras significativas en ambos grupos tanto en la potencia aeróbica (PA) como en la anaeróbica (PN) $(p=.00)$. En cuanto a la potencia aeróbica, a través del Test de Course Navette obtuvimos un promedio mayor en el GE (pretest $=26.04$; postest $=$ 28.36) que en el GC (pretest $=26.48$; postest $=27.11$ ). En cuanto a la potencia anaeróbica, con el Test de Burpee como instrumento, hubo un promedio mayor en el $\mathrm{GE}$ (pretest $=23.85$; postest $=26.25$ ) que en el GC (pretest $=23.80$; postest $=24.70$ ).
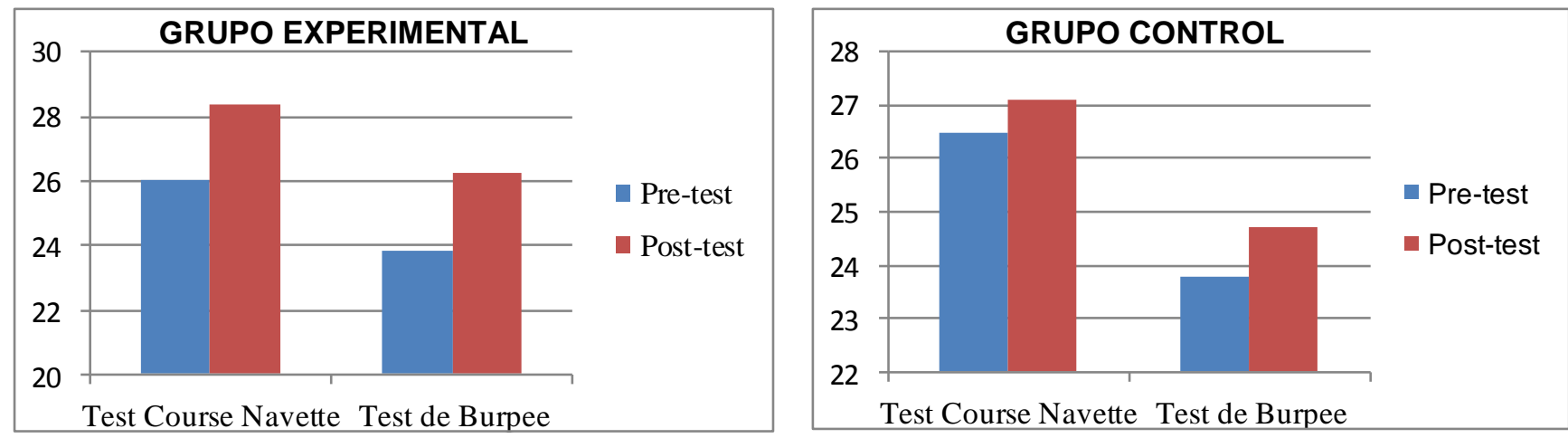

Figura 1. Evolución de la condición física (PA y PN) de ambos grupos en pre-test y post-test

\subsubsection{Esfuerzo percibido final del programa}

En cuanto al esfuerzo percibido final del programa de mejora de condición física, se obtuvieron diferencias significativas entre ambos grupos $(p=.00)$. El GE obtuvo puntuaciones menores $(M=11.45)$ con respecto a los que lo realizaron sin presencia de música $(M=15.05)$. Por lo tanto, los participantes que escucharon música durante la intervención percibieron un carácter de esfuerzo final inferior en comparación con aquellos que no escucharon música.

\subsubsection{Intención de seguir siendo activos}

En referencia a la intención de los sujetos de ser físicamente activos tras su paso por el programa de actividad física, a través del promedio de los ítems que forman el cuestionario (MIFA) se obtuvieron diferencias significativas $(p<.05)$ en ambos grupos. Los valores obtenidos del GE $(M=3.5)$ fueron mayores que los del $\mathrm{GC}(M=2.8)$. Por lo que los sujetos que han escuchado música durante el programa (GE) tienen una mayor intención a seguir con la práctica que los que no la escucharon (GC). 


\subsection{Efecto del tipo de música en variables psicológicas (GE)}

Teniendo en cuenta que en cada semana los sujetos escucharon música de distinto tempo musical, para la interpretación de los resultados de las siguientes variables se han agrupado las semanas en "semanas con música de tempo elevado (>100 bpm)" y "semanas con música de tempo bajo (<100 bpm)".

\subsubsection{Esfuerzo percibido durante las 5 semanas en función del tipo de música}

Se realizó el promedio de los resultados de las correspondientes semanas y se reflejaron diferencias significativas $(p<.05)$ en ambos tipos de música con respecto al carácter de esfuerzo percibido de los sujetos. Las semanas en las que se llevaron a cabo canciones con tempo musical elevado $\left(1^{\underline{a}}, 3^{\underline{a}}\right.$ y $\left.5^{\underline{a}}\right)$ obtuvieron puntuaciones inferiores $(M=11.16)$ con respecto a las que se escucharon canciones con tempo musical bajo (2 $2^{\underline{a}}$ y $\left.4^{a}\right)(M=13.72)$. Por lo que el carácter de esfuerzo percibido guarda una relación inversamente proporcional con el tempo musical de las canciones (ver Figura 2).

\subsubsection{Motivación en el ejercicio físico en función de la música escuchada}

En cuanto al grado en que la música motiva a los participantes, se apreciaron diferencias significativas $(p=.00)$ en la motivación en función del tempo de la música escuchada. Las semanas donde se emplearon canciones con tempo musical elevado $\left(1^{\underline{a}}, 3^{\underline{a}}\right.$ y $\left.5^{\mathrm{a}}\right)$ tuvieron puntuaciones superiores $(M=2.61)$ con respecto a las semanas $\left(2^{2}\right.$ y $\left.4^{a}\right)$ en las que se escucharon canciones con tempo musical bajo $(M=1.37)$. Por lo que observa que la motivación de los participantes guarda una relación directamente proporcional con el tempo musical de las canciones escuchadas (Figura 2).

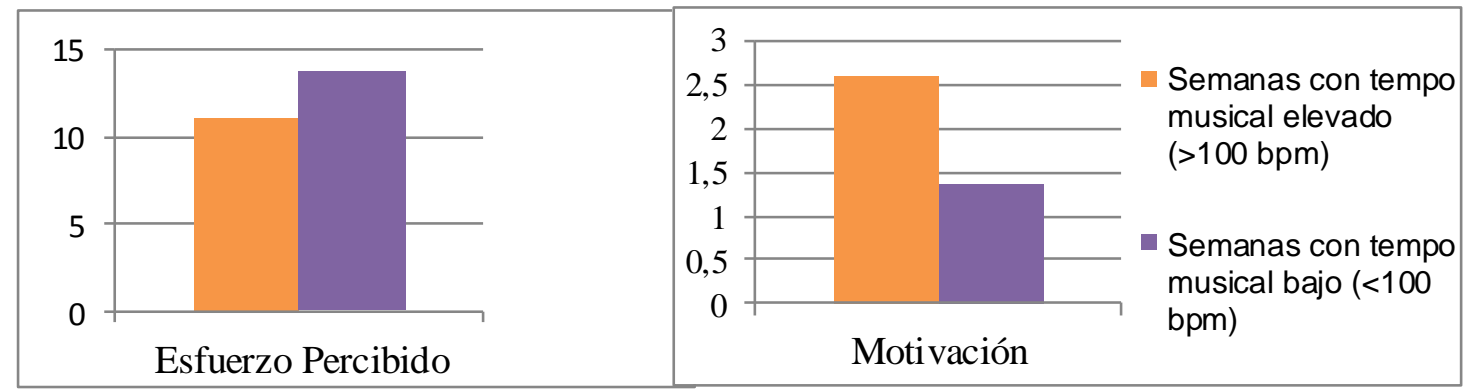

Figura 2. Comportamiento del carácter de esfuerzo percibido y la motivación en función del tempo musical de las canciones escuchadas en el grupo experimental (GE)

\subsubsection{Emociones manifestadas}

Se encontraron diferencias en los resultados de las emociones estudiadas en función del tipo de música (Figura 3). Por un lado, se obtuvieron valores elevados de emociones positivas (alegría, bienestar, confianza, felicidad) en canciones cuyo tempo musical fue elevado (>100 bpm). Por otro lado, algunas emociones negativas como el nerviosismo y la agresividad también presentaron elevadas puntuaciones con dicho tempo musical. Sin embargo, el resto de las emociones observadas (tristeza, debilidad/cansancio e indecisión) presentaron puntuaciones superiores en canciones con tempo musical bajo (<100 bpm) con respecto a las de elevado tempo. 


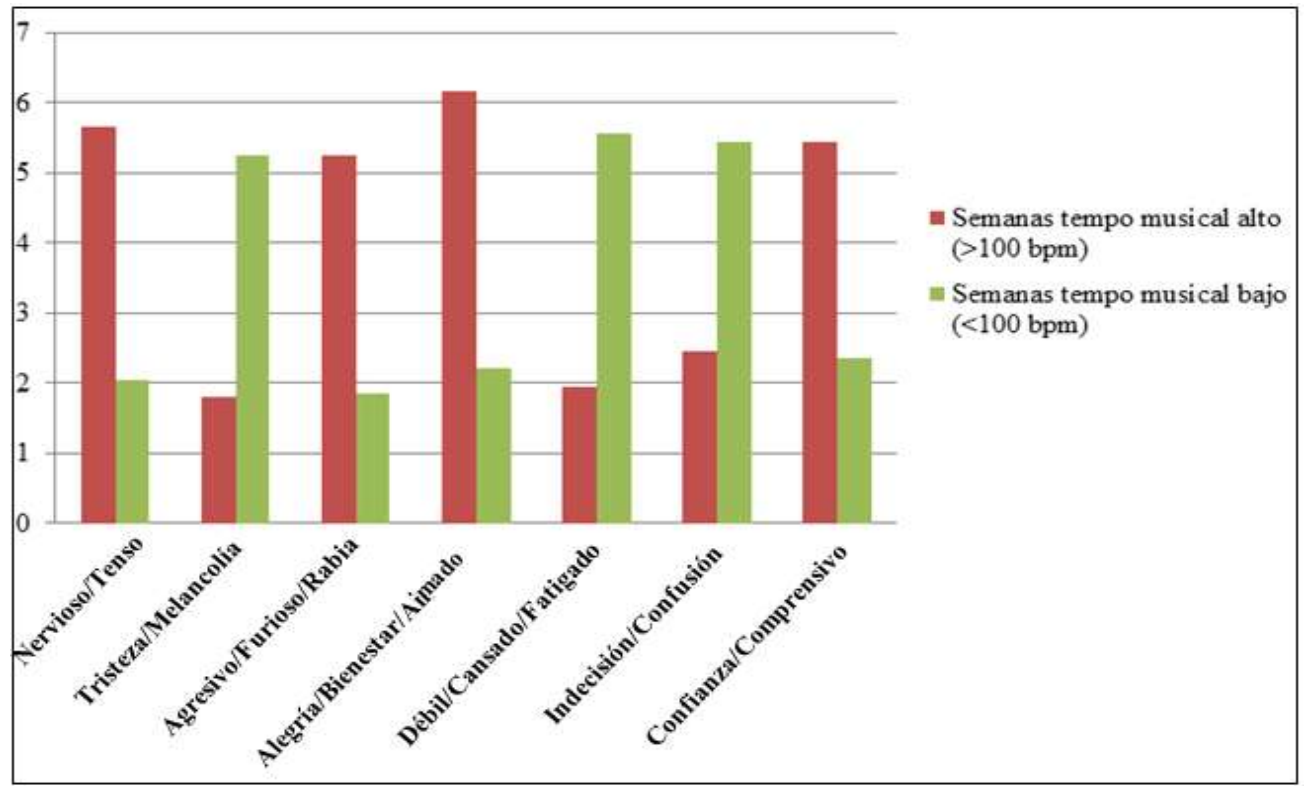

Figura 3. Gráfico comparativo de las emociones manifestadas en función del tempo musical de las canciones propuestas.

\section{DISCUSIÓN}

En el presente trabajo se evaluó la influencia de la música en la condición física de los participantes, en concreto focalizando el trabajo de la potencia aeróbica (PA) y anaeróbica (PN) a través de dos programas de actividad física similares; uno con presencia de música y otro sin ella. Coincidiendo con la línea de trabajo de Simpson y Karageorghis (2006) y Van der Vlist, Bartneck y Mäueler (2011), ambos grupos obtuvieron mejoras significativas en cuando a la PA y PN, siendo el grupo que escuchó música durante los ejercicios el que obtuvo mejoras más amplias. Esto podría ser posible debido a un aumento del trabajo físico submáximo cuando éste se sincroniza con el tempo de la música (Waterhouse, Hudson, y Edwards, 2010). Además, siguiendo este principio, sería conveniente el uso de música con tempo musical elevado (>100 bpm) para sincronizar los ejercicios a una mayor velocidad y así obtener mejoras más acentuadas en la condición física (CF). No obstante, en ocasiones esto puede resultar perjudicial para sujetos que no toleren cargas a dichas velocidades. Por lo que a raíz de esto y siguiendo el principio de individualización (Weineck, 2005), se propone la recomendación de canciones cuyo tempo musical se adapte a la velocidad de la carga que tolere el sujeto.

En cuanto al carácter de esfuerzo percibido, primero se analizó su comportamiento en los participantes de ambos programas una vez acabada la intervención (GE y GC) y segundo, se identificó su comportamiento en función del tipo de música escuchada durante la intervención. Por un lado, los sujetos del programa con presencia de música manifestaron un carácter de esfuerzo menor con respecto a los que no escucharon música. Por otro lado, el carácter de esfuerzo en las semanas con canciones de tempo musical elevado (>100 bpm) fue menor que en las semanas con canciones de tempo bajo (<100 bpm). En línea con las teorías de "percepción selectiva" de Broadbent (1958) y de "atención focalizada" de Hernández-Peon (1961), parace que el sistema nervioso centra la atención en la música como único estímulo, inhibiendo los demás envolventes durante la práctica deportiva y atenuando la sensación de cansancio (Copeland y Franks, 1991; Nideffer, 1976; Guillén y RuizAlfonso, 2013; Hernández-Peon, 1961; Ju-Han y Jing-Horng Lu, 2013; Pennebaker y 
Lightner, 1980; Rejeski, 1985). Por lo tanto, aunque la música no cambia la fatiga producida al realizar los ejercicios, sí cambia la percepción del sujeto acerca de ésta. Este efecto es mayor cuando la música presenta un tempo elevado (Karageorghis y Priest, 2012), más concretamente entre 100-130 bpm.

Asimismo, a través de los resultados obtenidos, existe una relación entre el tipo de música y las emociones manifestadas por parte de los participantes del GE. Si bien atendiendo a la literatura científica, más concretamente en Lipschultz y Chambliss (1992) y Karageorghis et al. (1996), en las canciones con tempo elevado denominadas como "música positiva-estimulante" se produjeron emociones positivas como bienestar, felicidad, satisfacción y confianza. Sin embargo, también se manifestaron emociones negativas como la agresividad y el nerviosismo. Pero no siempre las emociones afectan de la misma forma en el ejercicio físico, ya que atendiendo a la clasificación de Hanin (2003), las emociones positivas y negativas pueden ser funcionales o disfuncionales para el individuo en función el contexto donde se encuentre. En este sentido, emociones como la agresividad puede ser funcional si ésta facilita el desempeño del individuo en ejercicios de intensidad moderada-intensa como correr o salir en bicicleta, sin embargo, dicha emoción sería disfuncional en el contexto de una clase de Pilates.

Además, siguiendo con la línea de trabajo de Lipschultz y Chambliss (1992) y Karageorghis et al. (1996), en las canciones de tempo bajo denominadas como "música negativa-relajante" se manifestaron emociones negativas más asociadas a la tristeza, insatisfacción, desconfianza, indecisión y debilidad. Consideramos que dichas emociones carecen de utilidad o son disfuncionales (Hanin, 2003) en contextos relacionados con la actividad física y el deporte.

Hay ciertos indicios de que las emociones puedan influir en el carácter de esfuerzo percibido de los participantes. Según Szmedra y Bacharach (1998) algunos estados psicológicos evocados por la música durante el ejercicio como el bienestar, puede contribuir a la vasodilatación; lo que influiría en variables hemodinámicas, el aclaramiento de ácido láctico y por tanto la disminución del cortisol, ya que según John et al. (2010) es un indicador de emociones negativas. Por lo que las emociones manifestadas durante el ejercicio con presencia música pueden influir a nivel fisiológico, reduciendo a su vez la percepción del esfuerzo. No obstante, sería conveniente el uso de instrumentos específicos que midan directamente el comportamiento de las variables fisiológicas mencionadas (pulsímetros, glucómetros, analizadores de lactato, etc.).

En cuanto a la motivación, aunque esté considerada por Lazarus (2000) y Bisquerra (2000) como una emoción positiva más, fue considerada el análisis de dicha variable por separado. De igual manera, se han encontrado diferencias entre el tipo de música y el índice de motivación de los participantes. Karageorghis y Priest (2012) afirmaron que son 4 factores los que dotan de cualidades motivacionales a una pieza musical (tempo, musicalidad, impacto cultural y asociación extra-musical), no obstante recalcan el tempo como el más determinante. Por ello, esta intervención fue orientada a encontrar diferencias en la motivación de los participantes en función del tempo de la música. De modo que las canciones con tempo musical elevado (>100 bpm) presentaron puntuaciones mayores de motivación con respecto a las de tempo bajo (<100 bpm). Con esto, consideramos imprescindible la combinación de "música positiva-estimulante" (>100 bpm) y ejercicio físico para conseguir y mantener una motivación elevada durante la práctica de ejercicio físico y en consecuencia, promover el disfrute de la actividad. 
Por último, y en relación con la motivación, se analizó la influencia de la música en la adherencia de los sujetos que la presenciaron durante el programa (GE) en comparación con aquellos que lo realizaron sin música (GC). Los resultados mostraron valores superiores en los participantes del grupo experimental, por lo que coincidiendo con Gluch (1993), Mann (1979) y Karageorghis y Priest (2012), un programa de actividad física con presencia de música podría aumentar la adherencia ya que aumentaría la motivación de los participantes. Por último, la música es considerada por dichos autores como un elemento de disfrute para la realización de actividad física, lo que favorecería en la intencionalidad de las personas para mantenerse físicamente activas (Brooks y Brooks, 2010; Karageorghis y Terry, 1997).

Este estudio presenta algunas limitaciones, que deberán ser tenidas en cuenta a la hora de generalizar los resultados o en futuros estudios. Aunque se han obtenidos resultados satisfactorios en cuanto a la condición física y las variables psicológicas, sería conveniente estudiar la evolución de dichas variables en un período de tiempo más prolongado y con una muestra más amplia, con vistas a obtener datos más fiables sobre el proceso estudiado. Además, hay que considerar que no se realizó un análisis inter-grupo en el postest de los dos grupos (GE y GC) con respecto a los dos indicadores de la CF elegidos. Por lo que habrá que testar los resultados de este estudio con otros análisis y estadísticos, que nos aseguren de forma objetiva los efectos de la intervención.

Asimismo, aunque se ha medido la percepción del carácter de esfuerzo de los participantes, sería interesante obtener otra medición de la intensidad del esfuerzo teniendo en cuenta el comportamiento de variables como la frecuencia cardiaca en el ejercicio físico con/sin música.

Por último, una de las características que posee la música y que no se ha tenido en consideración ha sido la letra o el contenido verbal de las canciones. Por ello, sería conveniente analizar ésta como variable independiente, a fin de determinar sus posibles efectos sobre la preparación física y psicológica de los participantes.

\section{CONCLUSONES}

Los resultados de este estudio han mostrado la efectividad de programas de mejora de la condición física con música (GE) y sin música (GC). En concreto, en ambos grupos se encontraron mejoras significativas en las variables físicas medidas (potencia aeróbica y potencia anaeróbica), siendo más amplias en el grupo experimental (GE). Además, el comportamiento de las variables psicológicas fue condicionado por la música, mostrándose un menor carácter de esfuerzo, una mayor intención de seguir haciendo ejercicio y una mayor motivación en canciones con tempo elevado (> $100 \mathrm{bpm}$ ). Así pues, la música parece influir tanto en la preparación física como en aspectos psicológicos de los sujetos durante la práctica de ejercicio.

\section{REFERENCIAS}

Andrade, E., Arce, C., De Francisco, C., Torrado, J., y Garrido, J. (2013). Versión breve en español del cuestionario POMS para deportistas adultos y población general. Revista de Psicología del Deporte, 22(1), 95-102.

Anshel, M. H., y Marisi, D. Q. (1978). Effects of music and rhythm on physical performance. Research Quarterly, 49, 109-113.

Bisquerra, R. (2000). Educación emocional y bienestar. Barcelona: Praxis. 
Bonny, H. L. (1987). Music the language of immediacy. The Arts in Psychotherapy, 14, 255-261.

Borg, G. (1982). Psychophysical bases of perceived exertion. Journal of Medicine \& Science in Sports \& Exercise, 14(5), 377-381.

Broadbent, D. E. (1958). Perception and communication. Elmsford: Pergamon.

Brooks, K., y Brooks, K. (2010). Enhancing sport performance through the use of music. Journal of Exercise Physiology, 13(2), 52-57.

Bunt, L., y Stige, B. (2014). Music Therapy. An art beyond words. (2ª ed.). New York: Routledge.

Caballero-Meneses, J., y Menez, M. (2010). Influencia del tempo de la música en las emociones. Revista Colombiana de Psicología, 19(1), 37-44

Campos, J., y Ramón, V. (2006). Teoría y planificación del Entrenamiento Deportivo. Barcelona: Paidoribo.

Copeland, B., y Franks, D. (1991). Effects of types and intensities of background music on treadmill endurance. Journal of Sport Medicine and Physical Fitness, 31, 100-103.

Del Olmo, M. (2009). Musicoterapia con bebés de 0 a 6 meses en cuidados intensivos pediátricos (Tesis Doctoral). Madrid: Universidad Autónoma de Madrid, España.

Dueñas, O., y Herrera, M. (2015). Musicoterapia una alternativa para el tratamiento de la depresión en el Adulto Mayor. Ponencia Presentada en la I Convención Salud 2015. La Habana, Cuba. Recuperado de http://www.convencionsalud2015.sld.cu/index.php/convencionsalud/2015/paper /view/874/760

Elvira, M. A. (2004). Propuesta pedagógica para la utilización de la musicoterapia en la atención de niños que presentan dificultades de aprendizaje (Tesis de Grado). Universidad Nacional Abierta, Venezuela.

García, F. (2004). Herramientas psicológicas para entrenadores y deportistas. La Habana, Cuba: Editorial Deportes.

Gastón César García, G. C., y Secchi, J. D. (2014). Test Course Navette de 20 metros con etapas de un minuto. Una idea original que perdura hace 30 años. Apuntes: Medicina de L'Esport, 49(183), 93-103.

Gfeller, K. (1988). Musical components and styles preferred by young adults for aerobic fitness activities. Journal of Music Therapy, 25, 28-43.

Gluch, P. D. (1993). The use of music in preparing for sport performance. Contemporary Thought, 2, 33-53

Guillén, F., y Ruiz-Alfonso, Z. (2015) Influencia de la música en el rendimiento físico, esfuerzo percibido y motivación. Revista Internacional de Medicina y Ciencias de la Actividad Física y el Deporte, 15(60), 701-717

Hanin, Y. (2003). Performance related emotional states in sport: A qualitative analysis. Forum: Qualitative Social Research 4(1), 1-31.

Hayakawa, Y., Miki, H., Takada, K., y Tanaka, K. (2000). Effects of music on mood during bench stepping exercise. Perceptual and Motor Skills, 90, 307-314.

Hein, V., Müür, M., y Koka, A. (2004). Intention to be physically active after school graduation and its relationship to three types of intrinsic motivation. European Physical Education Review, 10(1), 5-19. 
Hernández-Peon, R. (1961). The efferent control of afferent signals entering the central nervous system. Annals of the New York Academy of Sciences, 89, 866-82.

John, S., Verma, S., y Khanna, G. (2010). The effect of music therapy on salivary cortisol as a reliable marker of pre-competition stress in shooting performance. Journal of Exercise Science and Physiotherapy, 6(2), 70-77.

Ju-Han, L., y Jing-Horng Lu, F. (2013). Interactive Effects of Visual and Auditory Intervention on Physical Performance and Perceived Effort. Journal of Sports Science and Medicine, 12(3), 388-393

Karageorghis, C. I. (2008). The scientific application of music in sport and exercise. London: Hodder Education.

Karageorghis, C. I., Drew, K. M., y Terry, P. C. (1996). Effects of pretest stimulative and sedative music on grip strength. Perceptual and Motor Skills, 83, 13471352.

Karageorghis, C. I., Terry, P., y Lane, A. (1999). Development and initial validation of an instrument to assess the motivational qualities of music in exercise and sport: The Brunel Music Rating Inventory. Journal of Sports Sciences, 17 (9), 713-724

Karageorghis, C. I., y Priest, D. (2012). Music in the exercise domain : a review and synthesis ( Part I ). International Review of Sport and Exercise Psychology, 5(1), 44-66.

Karageorghis, C. I., y Terry, P.C. (1999). Affective and psychophysical responses to asynchronous music during submaximal treadmill running. Proceedings of the European College of Sport Science Congress, 218). ECSS: Rome, Italy.

Karageorghis, C.I., y Terry, P.C. (1997). The psychophysical effects of music in sport and exercise: A review. Journal of Sport Behavior, 20, 54-68.

Larsen, R. J., y Buss, D. M. (2005). Enfoques fisiológicos de la personalidad. En R. J. Larsen y D. M. Buss (Eds.), Psicología de la personalidad (pp. 192-229). Madrid: Mc Graw Hill.

Lazarus, R. S. (2000). How emotions influence performance in competitive sports. The Sport Psychologist, 14, 229-252.

Lipschultz, W., y Chambliss, C. (1992). The effect of music and intensity on optimism. Ursinus College, 4(1), 43-49.

MacDougall, R. (1903). The affective quality of auditory rhythm in its relation to objective forms. Psychological Review, 10, 15-36.

Mandianes, M. (2003). Caracterización de la Religión Popular. En C. Álvarez, M. J. Buxó y S. Rodríguez (Eds.), La Religiosidad Popular (pp. 44-52). Barcelona: Anthropos Editorial.

Mann, M. (1979). Use and effect of music as an ergogenic aid. Rowing, 23, 19-23.

Montánchez, M. L. (2012). La influencias de la musicoterapia en el abordaje de las dificultades de aprendizaje. Revista Axioma, 2(9), 37-42.

Moreno, J. A., Moreno, R., y Cervelló, E. (2007). El autoconcepto físico como predictor de la intención de ser físicamente activo. Psicología y Salud, 17(2), 261-267.

Morgan, W. (1973). Psychological factors influencing perceived exertion. Journal of Medicine \& Science in Sports \& Exercise, 5(2),97-103. 
Murray, N., Sujan, H., Hirt, E. R., y Sujan, M. (1990). The influence of mood on categorization: A cognitive flexibility interpretation. Journal of Personality and Social Psychology, 59, 411-42

Nideffer, R. (1976). Test of attentional and interpersonal styles. Journal of Personality and Social Psychology, 34, 394-404.

Palomares, I. (2014). El equilibrio del cuerpo, la energía y las emociones a través del sonido: Biomúsica. Tesina Universidad de Jaén. Facultad de Humanidades y Ciencias de la Educación. Andalucía, España.

Pennebaker, J. W., y Lightner, J. M. (1980). Competition of internal and external information in an exercise setting. Journal of Personality and Social Psychology, 39(1), 165-74.

Pineda, E., y Pérez, Y. (2011). Musicoterapia aplicada a niños con síndrome de Down. Revista Cubana de Pediatría, 83(2), 42-148.

Reeve, J. (2003). Naturaleza de la emoción. En J. Reeve (Ed.), Motivación y emoción (pp. 441-478). Interamericana: Mcgraw-Hill.

Rejeski, W. J. (1985). Perceived exertion: An active or passive process? Journal of Sport Psychology, 75, 371-378.

Sánchez, S. (2010). Musicoterapia como recurso educativo para personas con discapacidad: parte I. Revista digital: Innovación y Experiencias Educativas, 37, 1-9. Recuperado de http://docplayer.es/15920374-Musicoterapia-como-recursoeducativo-para-personas-con-discapacidad-parte-i.html

Simpson, S. D., y Karageorghis, C. I. (2006). The effects of synchronous music on 400m sprint performance. Journal of Sports Sciences, 24, 1095-1102.

Szmedra, L., y Bacharach, D. W. (1998). Effect of music on perceived exertion, plasma lactate, norepinephrine and cardiovascular hemodynamics during treadmill running. International Journal of Sports Medicine, 19, 32-37.

Umemoto, T (1990). The Psychological Structure of Music. Music Perception: An Interdisciplinary Journal, 8(2), 115-127.

Uppal, A. K., y Datta, U. (1990). Cardiorespiratory response of junior high school girls to exercise performed with and without music. Journal of Physical Education and Sports Science, 2, 52-56.

Vallerand, R. J. (2001). A hierarchical model of intrinsic and extrinsic motivation in sport and exercise. En G.C. Roberts (Ed.), Advances in motivation in sport and exercise (pp. 263-319). Champaign, IL: Human Kinetics.

Van der Vlist B, Bartneck C, y Mäuler S (2011) Mo Beat: Using interactive music to guide and motivate users during aerobic exercising. Applied Psychophysiology and Biofeedback, 36, 135-145.

Waterhouse, J., Hudson, P., y Edwards, B. (2010). Effects of music tempo upon submaximal cycling performance. Scandinavian Journal of Medicine \& Science in Sports, 20(4), 662-669.

Weineck, J. (2005). Entrenamiento Total (primera edición). Barcelona: Paidotribo

\section{Agradecimientos}

Este artículo es fruto de mi trabajo fin de grado (TFG) y quiero agradecer la inestimable ayuda y colaboración de mi tutor, el Dr. Pedro Sáenz-López Buñuel. 